[0212-7199 (2005) 22: 5; pp 217-221] ANALES DE MEDICINA INTERNA Copyright $(2005$ ARAN EDICIONES, S.L

AN. MED. INTERNA (Madrid) Vol. 22, N. ${ }^{\circ}$ 5, pp. 217-221, 2005

\section{Estudio de las bacteriemias en el Servicio de Medicina Interna de un hospital de grupo 2. Análisis de los tres últimos años}

\author{
A. I. FRANCO MORENO, S. CASALLO BLANCO, F. MARCOS SÁNCHEZ, \\ M. SÁNCHEZ CASADO ${ }^{1}$, M. T. GIL RUIZ ${ }^{2}$, A M. MARTÍNEZ DE LA CASA \\ MUÑOZ
}

Servicios de Medicina Interna, ${ }^{1}$ Medicina Intensiva, ${ }^{2}$ Microbiología $y{ }^{3} \mathrm{MFyC}$. Hospital

Nuestra Señora del Prado. Talavera de la Reina. Toledo

STUDY ON BACTEREMIA IN THE SERVICE OF INTERNAL MEDICINE OF A GROUP 2 HOSPITAL. ANALYSIS OF RECENT THREE $Y E A R S$

\section{RESUMEN}

Introducción: La bacteriemia sigue siendo uno de los principales problemas de la patología infecciosa. El interés de realizar este estudio ha sido conocer el modelo de presentación y desarrollo de la bacteriemia en nuestro medio, con el propósito de prevenir y tratar mejor esta entidad.

Pacientes y método: Se trata de un estudio retrospectivo, sin ningún tipo de intervención, de las bacteriemias significativas detectadas en el servicio de Medicina Interna de un hospital secundario durante tres años. Durante el periodo de estudio, 1 de enero de 2001 hasta el 31 de diciembre de 2003, se procesó en el Servicio de Microbiología Clínica 4.719 hemocultivos (HC), de los que 1964 (41,6\%) fueron remitidos desde el servicio de Medicina Interna. Resultaron positivos $336(17,1 \%)$ de los que $81(24,1 \%)$ correspondieron a episodios de bacteriemia verdadera, y $255(75,9 \%)$ se consideraron contaminaciones.

Resultados: En total se estudiaron 81 episodios de bacteriemia verdadera correspondientes a 77 pacientes (sólo 4 pacientes presentaron dos episodios de bacteriemia). Se calculó una tasa de incidencia de 28.25 episodios por cada mil ingresos hospitalarios. La edad media fue de 72 años (IC 95\%: 68,62-75,38). Predominaron los pacientes varones $(51,9 \%)$ mayores de 60 años. El lugar de adquisición de la bacteriemia ha sido el medio extrahospitalario en el $75,3 \%$ de los casos y el nosocomial en el $24,7 \%$. Las enfermedades de base más frecuentes fueron la hipertensión arterial y la diabetes. Las manipulaciones genitourinarias y vasculares fueron las más relacionadas con desarrollo de bacteriemia. Como foco de origen destacan por su frecuencia: nefrourológico $(42.0 \%)$, respiratorio $(19.8 \%)$ y abdominal $(13,6 \%)$. En nuestro medio los patógenos más frecuentemente aislados han sido Escherichia coli $(33,0 \%)$ y Staphylococcus coagulasa negativo $(15,9 \%)$. El tratamiento antibiótico empírico fue correcto en el $86,2 \%$ de los casos, siendo el grupo de cefalosporinas de tercera generación el más utilizado. La mortalidad global fue del $16 \%$ (13 pacientes), y la directamente relacionada con la bacteriemia del $61 \%$.

Conclusiones: Destacamos una incidencia elevada de episodios de bacteriemia en comparación con otras series de centros de similares características. Hemos obtenido un porcentaje significativo de episodios por Staphylococcus coagulasa negativo como se viene analizando los últimos años, lo que nos obliga a una valoración minuciosa de los posibles contaminantes y al entrenamiento del personal sanitario en la técnica de extracción.

PALABRAS CLAVE: Bacteriemia. Mortalidad. Infección nosocomial.

\section{ABSTRACT}

Introduction: Bacteremia remains one of the most relevant problems in infectious disease. The interest of this study was to know the presentation and development pattern of bacteremia in our environment, in order to best prevent and treat this entity.

Patients and method: A retrospective, no interventional study, on significant bacteremia detected in the Service of Internal Medicine of a secondary level hospital over three years was carried out. Through the study length, from January 12001 until December 31 2003, 4,719 blood cultures were processed by the Service of Microbiology; of these, 1964 $(41.6 \%)$ were submitted by the Service of Internal Medicine. Results were positive in $336(17.1 \%)$; of these, 18 (24.1\%) correlated with episodes of true bacteremia, and $255(75.9 \%)$ were deemed as contaminations.

Results: Overall, 81 episodes of true bacteremia were studied, from 77 patients (4 patients presented with 2 episodes). An incidence rate of 28.25 episodes per 1000 hospital admissions was estimated. Mean age was 72 years (95\% CI: 68.62-75.38). Males over 60 years-old were predominant $(51.9 \%)$. Bacteremia was community-adquired in $75.3 \%$ of cases, and nosocomial in $24.7 \%$. Commonest baseline diseases were elevated arterial blood pressure and diabetes mellitus. Bacteremia development was mostly related to genitourinary and vascular handling. Most of them were nephrourological (42.0\%), respiratory (19.8\%) and abdominal $(13.6 \%)$ in origin. In our environment, Escherichia coli $(33.0 \%)$ and Staphylococcus coagulase-negative (15.9\%) were the most commonly isolated pathogens. Empiric antibiotic therapy was correct in $86.2 \%$ of cases; third generation cephalosporins were the most used. Overall mortality rate was $16 \%$ (13 patients), and bacteraemia-related mortality was $61 \%$.

Conclusions: A high incidence of bacteremia episodies is noteworthy, as compared with series from other centers. The percentaje of episodes due to Sthapylococcus coagulase-negative was significant, as it is the rule in recent years; thus, a thoroughly assessment of potential contaminants, as well as staff training in drawing techniques becomes necessary.

KEY WORDS: Bacteremia. Mortality. Nosocomial infection.

Franco Moreno AI, Casallo Blanco S, Marcos Sánchez F, Sánchez Casado M, Gil Ruiz MT, Martínez de la Casa Muñoz AM. Estudio de las bacteriemias en el Servicio de Medicina Interna de un hospital de grupo 2. Análisis de los tres últimos años. An Med Interna (Madrid) 2005; 22: $217-221$ 


\section{INTRODUCCIÓN}

Las primeras descripciones de bacteriemia se realizaron a mediados de este siglo, observándose en la década de los 90 un aumento de la incidencia de bacteriemias significativas junto con cambios clínicos y epidemiológicos de las mismas, todo ello condicionado por un aumento de procedimientos invasivos, uso de materiales protésicos, accesos intra-vasculares, aparición de procesos patológicos (SIDA) relacionados con un estado de inmunodepresión severa y tratamientos antibióticos de amplio espectro prolongados (1-3).

Los estudios sobre septicemias estiman actualmente una incidencia entre 3-30 episodios por 1.000 ingresos hospitalarios y año, con una mortalidad variable entre el 30 y $40 \%$ $(3,4)$.

El objetivo de este estudio se sustenta en la necesidad de conocer la realidad clínico-microbiológica de nuestro medio e identificar aquellas variables potencialmente tratables que condicionan el pronóstico en la población con bacteriemia.

\section{MATERIAL Y MÉTODO}

El Hospital Nuestra Señora del Prado es un hospital de segundo nivel con 268 camas y una cobertura para 137.000 habitantes. Se estudiaron de forma retrospectiva todos los pacientes ingresados en el Servicio de Medicina Interna con hemocultivos positivos durante el periodo de tiempo comprendido entre el 1 de enero de 2001 y el 31 de diciembre de 2003. Se diseñó un protocolo previo para la recogida de datos donde se analizaron 10 variables categorizadas como clínicoepidemiológicos, factores de riesgo intrínseco y extrínseco, microbiológicas, evolución y supervivencia (Tabla I). Las fuentes de información han sido el laboratorio de Microbiología Clínica, que ha proporcionado los resultados positivos de los hemocultivos procesados, y la historia clínica del paciente. El análisis estadístico se realizó basándose en el programa informático SPSS.

\section{DEFINICIONES GENERALES} ción.

1. Episodio de bacteriemia verdadera (BV) y contamina-

Se consideraron bacteriemias verdaderas basándose en el número de "set" positivos, los datos clínicos acompañantes, las propias características de los microorganismos y si éstos aparecían en otros focos. Se consideró el mismo episodio de bacteriemia cuando se aislaron los mismos microorganismos durante los primeros siete días, ocasionalmente, periodos más largos fueron considerados si la situación clínica así lo indicaba.

\section{Adquisición.}

Se consideró intrahospitalaria cuando el episodio de BV se desarrolló después de las 48 horas de estancia en el hospital o si estuvo claramente relacionado con algún procedimiento diagnóstico o terapéutico que se practicara después del ingreso.

3. Factores de riesgo.

-Factores de riesgo intrínseco o enfermedad de base

Se consideró de manera individual basándose en los criterios de McCabe y Jackson:

\section{TABLA I}

VARIABLES A ESTUDIO

Sexo
Edad

$<40$ años

40-60 años

$>60$ años

Tipo de infección

Nosocomial/comunitaria

Foco de origen

Abdominal Respiratorio

Endocardio Nefrourológico

SNC

Osteoarticular Piel y partes blandas

Desconocido

Enfermedad de base (factores de riesgo intrínsecos)

$\begin{array}{ll}\text { Cirrosis hepática } & \text { ADVP } \\ \text { Enfermedad hematológica } & \text { LCFA } \\ \text { Diabetes Mellitus } & \text { VIH } \\ \text { Insuficiencia renal crónica } & \text { HTA } \\ \text { Tumores sólidos } & \text { Neutropenia }\end{array}$

Factores de riesgo extrínsecos

Procedimientos invasivos

Antibioterapia

Citostáticos/radioterapia

Corticoides

Germen aislado

Tto. empírico No valorable/correcto/incorrecto

Evolución Curación/exitus (relacionado,no relacionado)

- Enfermedad hematológica.

- Tumores sólidos.

- Cirrosis hepática.

- HTA (hipertensión arterial).

- LCFA (limitación crónica al flujo aéreo).

- ADVP (adictos a drogas por vía parenteral).

- Diabetes mellitus.

- Insuficiencia renal crónica.

- VIH (linfocitos CD4 <500).

- Neutropenia (recuento de granulocitos igual o inferior a $1.000 / \mathrm{mm}^{3}$.

-Factores de riesgo extrínseco o predisponentes.

Dentro de los mismos se consideraron todas aquellas manipulaciones realizadas al menos diez días antes del desarrollo de la bacteriemia. Estas incluyeron manipulaciones genitourinarias, quirúrgicas, respiratorias, digestivas, vasculares y traumatológicas. También se incluye en este apartado el tratamiento previo con antibióticos a dosis terapéuticas durante al menos los siete días previos. De igual manera se consideró el empleo de citostáticos o radioterapia. Por otro lado se tuvo en cuenta el empleo de corticoides a dosis igual o superior a $20 \mathrm{mg} /$ día de prednisona de forma diaria, al menos una semana antes del desarrollo de la bacteriemia.

4. Foco de origen.

La determinación del foco de origen se basó en hallazgos clínicos y/o microbiológicos. Cuando no se halló ninguna 
localización o si los datos fueron confusos se determinó como de origen desconocido.

5. Tratamiento antibiótico.

Se define tratamiento empírico el realizado tras la extracción del hemocultivo y hasta la llegada del antibiograma. Tratamiento específico es aquel realizado según antibiograma. En este apartado se utilizaron las siguientes definiciones

- No valorable, cuando el paciente estaba con tratamiento antibiótico por otro proceso infeccioso, antibiótico cuya actividad no fue demostrada "in vitro", o se produzco el fallecimiento del paciente antes de instaurar el tratamiento.

- Correcto, cuando se demostró su actividad "in vitro" frente al o los microorganismos aislados y se administró durante un periodo de tiempo no superior a 48 horas. También se consideró correcto en las asociaciones de antibióticos cuando al menos uno de éstos era activo.

- Incorrecto, la ausencia de utilización de antibióticos, su uso a dosis infraterapeúticas, administración posterior a 48 horas y no realizar tratamiento quirúrgico cuando este estaba indicado.

6. Evolución.

En relación con la evolución se distinguió: curación, muerte directamente relacionada con la bacteriemia y muerte indirectamente relacionada con la misma. Se definió curación la ausencia de signos clínicos de infección al alta hospitalaria, muerte directamente relacionada con la bacteriemia la producida en la primera semana posterior al desarrollo de la misma cuando los datos clínicos así lo apoyaban, y muerte indirectamente relacionada la concurrencia de cualquier proceso no relacionado con la misma.

\section{MÉTODO ESTADÍSTICO}

Las variables cuantitativas se han expresado mediante la media (desviación estándar) y cuando no han seguido una distribución normal mediante la mediana. Los estadísticos empleados para las variables cualitativas han sido los porcentajes, expresando entre paréntesis el número de casos. Las pruebas de asociación empleadas para las variables cualitativas han sido la chi cuadrado, utilizándose la prueba exacta de Fisher cuando no se cumplían las condiciones de aplicación. Para estudiar las variables cuantitativas hemos utilizado la prueba paramétrica de U Mann-White. Se consideró significativa con un valor de $\mathrm{p}<0,05$.

\section{RESULTADOS}

\section{EPIDEMIOLOGÍA (Tabla II)}

En el periodo de estudio se detectaron 81 episodios de bacteriemia verdadera significativa en 77 pacientes con una incidencia de 28,25 casos/1.000 admisiones. La edad media fue de 72 años (IC95\% 68,6-75,3), con predominio del sexo masculino $(51,9 \%)$, aunque las diferencias no fueron significativas. La mortalidad total fue del $16 \%$, mientras que directamente atribuida a la bacteriemia fue del $61,5 \%$. La estancia media fue de 15.6 días (IC95\% 13,4-17,7).

La enfermedad de base se presentó en el 81,5\% de pacientes. La HTA y la DM fueron las mas frecuentes, presentes en casi la mitad de pacientes (Fig. 1). El 16\% eran portadores de

\section{TABLA II}

ANÁLISIS DESCRIPTIVO DE LAS VARIABLES CLÍNICOEPIDEMIOLÓGICAS

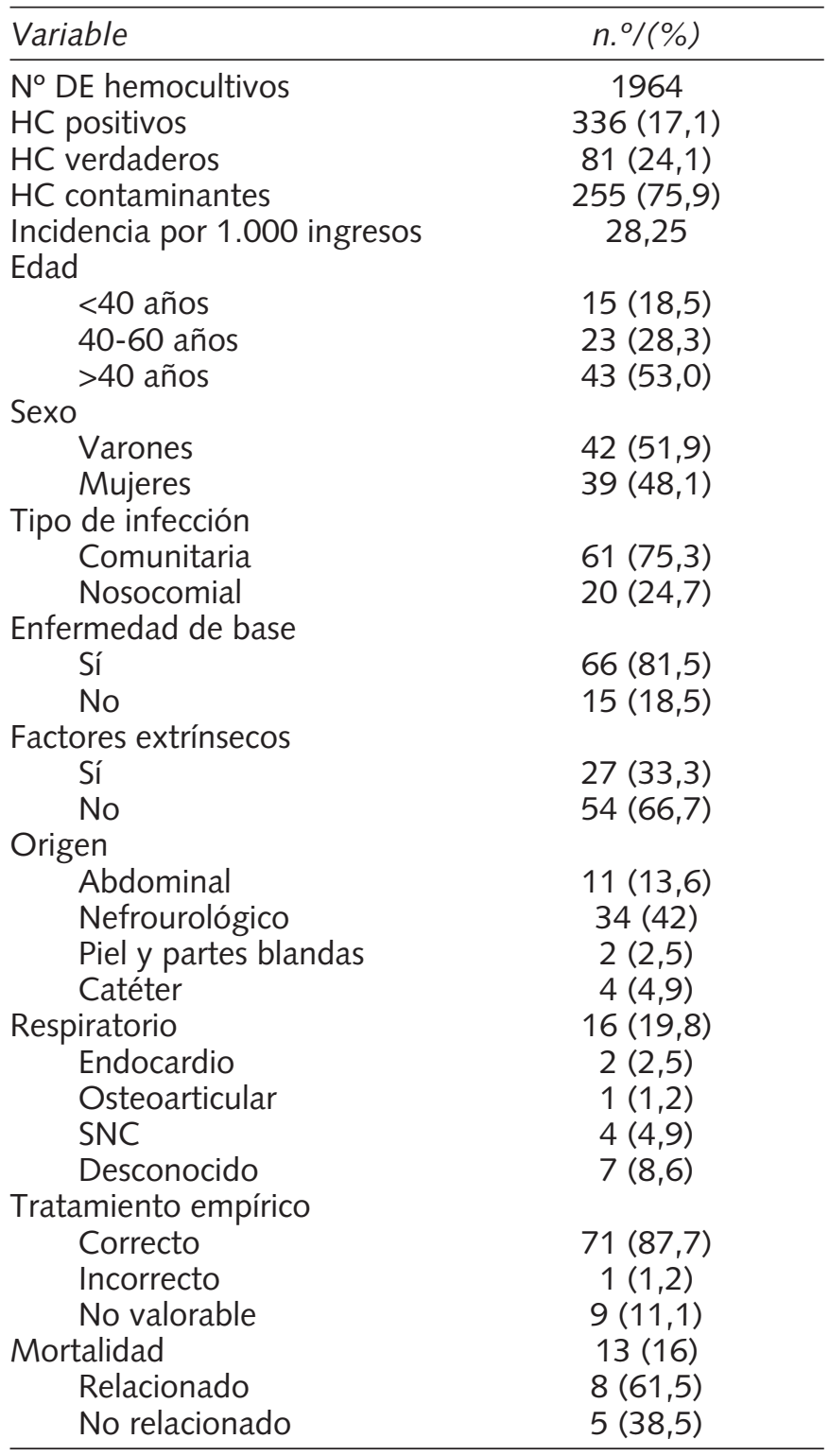

neoplasia o imnunosupresión, en este grupo el riesgo de muerte fue mayor pero no en términos de significación estadística.

El primer foco de origen fue el urinario $(42 \%)$, seguido del respiratorio $(19,8 \%)$ y abdominal $(13,6 \%)$, y hasta en un $8,6 \%$ de los casos el origen fue desconocido (Fig. 2). En este último grupo predominó el origen nosocomial. En un $66,7 \%$ de los aislamientos no se identificaron factores predisponentes, de ellos predominaron las manipulaciones genitourinarias y vasculares (Fig. 3).

\section{DATOS MICROBIOLÓGICOS (Tabla III)}

El origen nosocomial ocupó el 24,7\% de casos y el comunitario el 75,3\%. Existió mayor mortalidad en el grupo nosocomial, alcanzando significación estadística $(\mathrm{p}<0,032)$. La 


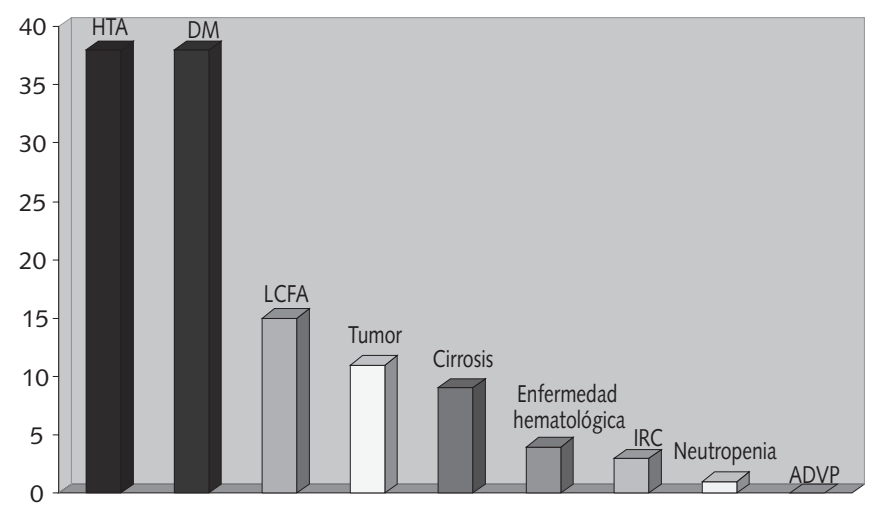

Fig. 1. Factores intrínsecos o enfermedades de base.

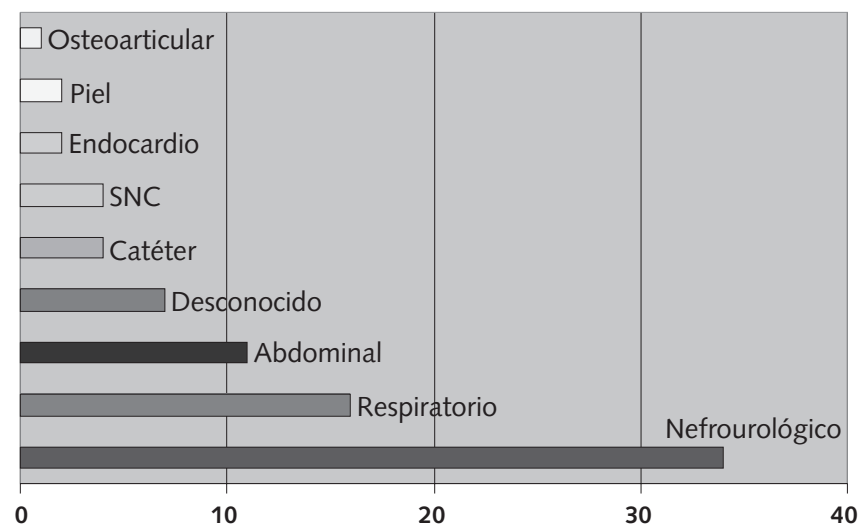

Fig. 2. Foco de origen.

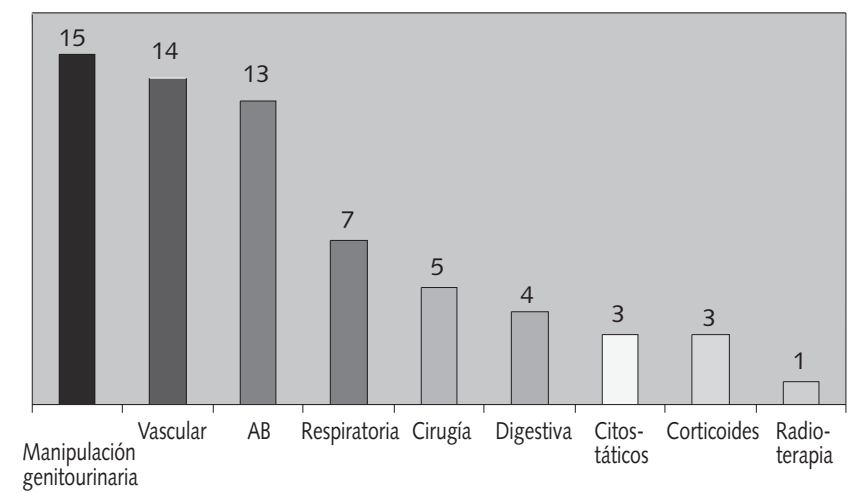

Fig. 3. Factores extrínsecos o predisponentes.

infección fue monomicrobiana en el 91,4\% de casos y polimicrobiana en el 8,6\%. Existió un ligero predominio de los Gram (-), siendo los principales aislamientos de Escherichia coli $(33,3 \%)$ y los Staphylococcus coagulasa negativo $(15,9 \%)$. Se aisló un caso de Candida spp. y otro de Clostridium spp. Atendiendo al tipo de patógeno no se identificaron diferencias en cuanto a mortalidad.
TABLA IIII

ANÁLISIS DESCRIPTIVO DE LOS DATOS MICROBIOLÓGICOS

\begin{tabular}{lc}
\hline Variable & $n . \%(\%)$ \\
\hline Polimicrobiana & $7(8,6)$ \\
Monomicrobiana & $74(91,4)$ \\
Escherichia coli & $27(33,3)$ \\
Staphylococcus coagulasa negativo & $12(15,9)$ \\
Streptococcus pneumoniae & $10(12,3)$ \\
Pseudomonas spp. & $9(11,1)$ \\
Enterococcus & $6(7.4)$ \\
Proteus spp. & $4(4,9)$ \\
Staphylococcus aureus & $3(3,7)$ \\
Salmonella spp. & $3(3,7)$ \\
Enterobacter spp. & $2(2,5)$ \\
Acinetobacter baumanii & $2(2,5)$ \\
Neisseria meningitidis & $1(1,2)$ \\
Otros & $2(2,4)$
\end{tabular}

\section{TRATAMIENTO}

El 100\% de los pacientes recibieron tratamiento antibiótico, un $87,7 \%$ recibió tratamiento adecuado, no existiendo diferencias en cuanto a mortalidad respecto a los que no lo recibieron. Al analizar el tiempo de supervivencia desde la bacteriemia documentada (Fig. 4), se objetivo que el mayor riesgo de muerte se produjo en los diez primeros días, dato que obtuvo significación estadística $(\mathrm{p}<0,001)$.

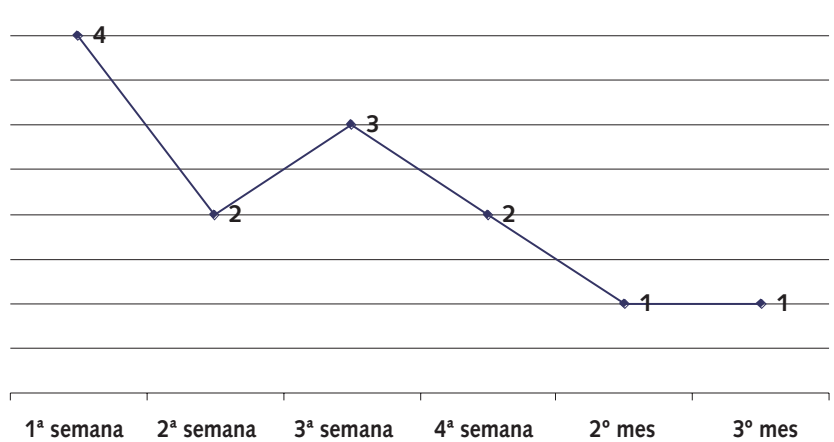

Fig. 4. Mortalidad y días de estancia.

\section{DISCUSIÓN}

La alta tecnificación de la medicina actual que conlleva: la implantación de materiales protésicos y el uso de accesos intravasculares, la aparición de nuevas enfermedades, avances en el campo del tratamiento definidos por el uso de terapias altamente agresivas y el empleo de antibioterapia de amplio espectro, han condicionado cambios en la flora microbiana intra y extrahospitalaria (5-7).

Un hecho muy relevante en nuestro medio es el envejecimiento de la población que obliga al ingreso en instituciones cerradas de un porcentaje importante de la población (8-11). 
Todo ello condiciona un aumento de enfermedades potencialmente graves y de "aitrogenia" que predispone a la adquisición de enfermedades infecciosas. Somos conscientes del contexto poblacional de nuestro medio, un centro de nivel secundario.

Hemos obtenido una incidencia que consideramos alta en comparación con otras series, dato puede explicarse por las características de nuestro centro (Medicina Interna acoge un porcentaje importante de pacientes procedentes de instituciones cerradas y de la Unidad de Vigilancia Intensiva). Los resultados obtenidos en cuanto a mortalidad global se equiparan a otros estudios, e incluso podríamos considerarla menor, dato que nos llama la atención considerando la ancianidad de la población a estudio. Como clásicamente la bacteriemia más frecuente ha sido la producida por la Escherichia coli de origen urinario. En nuestro estudio, como en las revisiones más recientes de otras series, se observa el creciente papel del grupo $S$. coagulasa negativo, lo que obliga a una minuciosa valoración de los presumibles contaminantes $(11,12)$. Aun así parece necesario la estandarización de técnicas de procesamientos de catéteres y la necesidad de protocolizar pautas de actuación en este tipo de infecciones $(12,13)$.
Se confirmó en nuestra serie un ligero predominio de los Gram (-) $(59,2 \%)$, a diferencia de lo que recogen otras series, no detectándose diferencias significativas en cuanto a mortalidad comparando ambos grupos.

No detectamos diferencias significativas en cuanto a la mortalidad en pacientes bacteriémicos según la enfermedad de base y la existencia de factores predisponentes, por lo que deben investigarse otros factores independientes no analizados en este trabajo.

En general se considera que el origen nosocomial de las infecciones es un factor de mal pronóstico, dato que se confirma en nuestra experiencia y que coincide con la mayoría de las series (14).

El análisis del tratamiento revela que consideramos la antibioterapia primordial, de forma que todos los pacientes recibieron antimicrobianos en las primeras 48 horas. El tratamiento empírico fue correcto en el $87,7 \%$ con predominio del grupo cefalosporinas y aminoglicósidos. Al analizar el tiempo de supervivencia desde la bacteriemia documentada, hemos objetivado que el mayor riesgo de muerte se produce en la primera semana, lo que corrobora la idea clásica que las complicaciones directas de la bacteriemia son de carácter precoz $(14,15)$.

\section{Bibliografía}

1. Jaimes F, Arango C, Ruiz G, Cuervo J, Botero J, Velez G, Upegui N, Machado F. Predicting bacteremia at the bedside. Clin Infect Dis 2004; 38: 357-62.

2. Metersky ML, Ma A, Bratzler DW, Houck PM. Predicting bacteremia in patients with community-acquired pneumonia. Am J Respir Crit Care Med 2004; 169: 342-7.

3. Pittet D, Li N, Woolson RF, Wenzel RP. Microbiological factors influencing the outcome of nosocomial bloodstream infections: A 6 year validated population based model. Clin Infect Dis 1997; 24: 106878

4. Pittet D, Li N, Wenzel RP. Association of secondary and polymicrobial nosocomial boodstream infections with higher mortality. Eur J Clin Microbiol Infect Dis 1993; 12: 813-9.

5. Noguerado A, Ruiz Giardín JM, Pizarro A, Méndez J, La Hulla F, Fernández M, et al. Análisis de factores pronósticos de mortalidad de las bacteriemias y fungemias en un hospital universitario. Evolución en 10 años. Rev Clin Esp 2001; 201: 122-9.

6. Grupo de Estudio de la Bacteriemia (Coordinador: F. Martinez-Luengas). Bacteriemia en seis hospitales españoles. Med Clin (Barc)1986; 86: 221-32.

7. Ruiz-Giardín JM, Noguerado A. Bacteriemia por anaerobios: características clinicoepidemiológicas de las bacteriemias por anaerobios en dos periodos con una diferencia de 10 años. An Med Interna (Madrid) 2004; 21: 425-32.

8. Weinstein MP, Towns ML, Quartety SM, Mirrett S, Reimer LG, Parmigiani $\mathrm{G}$, et al. The clinical significance of positive blood cultures in the 1990s: A prospective comprehensive evaluation of the microbiology, epidemiology, and outcome of bacteremia and fungemia in adults. Clin Infect Dis 1997; 24: 584-602.

9. Valles J, León C, Álvarez-Lerma F. Nosocomial bacteremia in critically ill patients: A multicenter study evaluating epidemiology and prognosis Spanish Collaborative Group for Infections in Intensive Care Units of Sociedad Española de Medicina Intensiva y Unidades Coronarias (SEMIUC). Clin Infect Dis 1997; 24: 387-95.

10. Haug JB, Harthug S, Kalager T, Digranes A, Solberg CO. Bloodstream infections at a Norwegian university hospital, 1974-1979 and 19881989: Changing etiology, clinical features, and outcome. Clin Infect Dis 1994; 19: 246-56.

11. García MA, López JJ, Moya R, Colmenero JD. Bateriemia neumocócica en el adulto en un hospital de tercer nivel. An Med Interna (Madrid) 2003; 20: 563-8

12. Deulofeu F, Cervello B, Capell S, Marti C, Mercade V. Predictors of mortality in patients with bacteremia: The importance of functional status. J Am Geriatr Soc 1998; 46: 14-8.

13. Dupont HL L, Spink WW. Infections due to Gram negative organims: An analysis of 860 patients with bacteriemia at the University of Minnesota Medical Center,1958-1966. Medicine 1969; 48: 307-32.

14. Bouza E, Perez-Molina J, Muñoz P. Cooperative Group of the European Study Group on Nosocomial Infections (ESGNI). Report of ESGNI-00 and ESGNI-002 studies. Bloodstream infections in Europe. Clin Microbiol Infect 1999; 5: 212-12.

15. Pazos R, Fernández R, Tinajas A, Nanín C, Bustillo M, Paz I, et al. Estudio de la sensibilidad antibiótica en las bacteriemias de un hospital secundario. An Med Interna (Madrid) 2004; 21: 483-87. 